\title{
Performance evaluation and analysis of four waves mixing in DWDM optical communications
}

\section{Ebrahim E. Elsayed}

Electronics and Communications Engineering Department, Faculty of Engineering, Mansoura University, Mansoura 35516, El-Dakahilia Governorate, Egypt.

Author's email address: engebrahem16@gmail.com

\section{i. Abstract}

Optical nonlinearities give rise to many ubiquitous effects in optical fibres '. These effects are interesting in themselves and can be detrimental in optical communication. In the Dense Wave length division multiplexing system (DWDM) the nonlinear effects plays important role .DWDM system offers component reliability, system availability and system margin. DWDM system carries different channels. Hence power level carried by fiber increases which generates nonlinear effect such as SPM ,XPM, SRS, SBS and FWM. Four wave mixing (FWM) is one of the most troubling issues. The FWM gives crosstalk in DWDM system whose channel spacing is narrow. Wavelength exchanging enables data swapping between two different wavelengths simultaneously. These phenomena have been used in many applications in Wavelength Division Multiplexing (WDM) optical networks such as, wavelength conversion, wavelength sampling, optical 3R, optical interconnects and optical add-drop multiplexing.

KEYWORDS: FWM, DWDM system, channel spacing, Optical spectrum, XPM, SRS, effect of dispersion.

\section{ii. INTRODUCTION}

FWM (Four Wave Mixing) or Four Photon Mixing (FPM) is the process whereby optical power from one channel in a multi-channel system is spilled over into adjacent channels. Three waves mix together to produce the fourth wave which may coincide with the original channel or may not be coinciding [1-8].

$f_{i j k}=f_{i} \pm f_{j} \pm f_{k}$

Equ.1 [16]

Total FWM products $=\frac{N^{2}(N-1)}{2}$.

Equ.2[16]

Where $\mathrm{N}$ is the no. of wavelengths.

Equation (1) shows the formula for the generation of FWM interfering term.

Equation (2) shows the total no. of interfering terms. 
- Newly formed FWM products

\# May fall on the original signal (cannot be filtered out).

May not fall on the original signal (can be filtered out).

- Spurious components are created, causing

- Interference

- Degradation of signals

- Cross talk
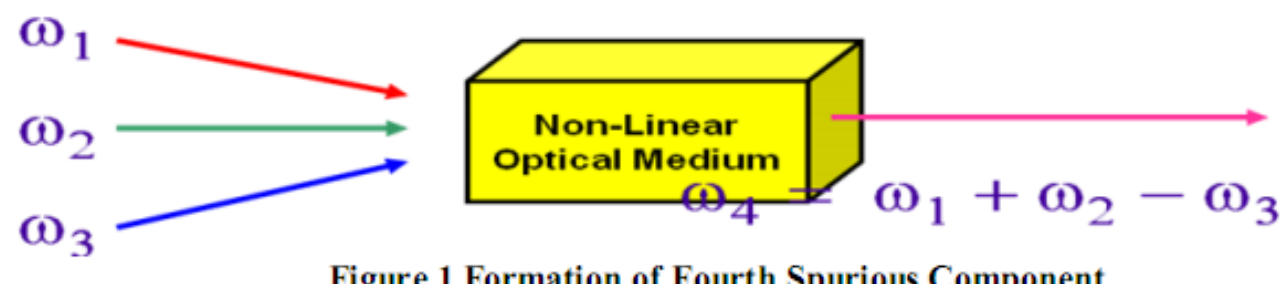

Figure 1 Formation of Fourth Spurious Component

Four-wave mixing transfers' energy from a strong pump wave to two waves up shifted and downshifted in frequency from the pump frequency $\omega 1$ [8-14] as shown in Fig. (1). If only the pump wave is incident at the fibre, and the phase matching condition is satisfied, the Stokes and anti-Stokes waves at the frequencies $\omega 3$ and $\omega 4$ can be generated from noise. On the other hand, if a weak signal at $\omega 3$ is also launched into the fibre together with the pump, the signal is amplified while a new wave at $\omega 4$ is generated simultaneously. The gain responsible for such amplification is called the parametric gain [4, 12-16].

\section{iii. Effects of FWM}

Four wave mixing (FWM) is one of the most troubling issues. Three signals combine to form a fourth spurious or mixing component, hence the name four waves mixing. Spurious components cause following problems [16-20]:

* Interference between wanted signal(cross)

* It generates additional noise and degrades system performance

* Power is lost from wanted signals into unwanted spurious signals

FWM can be substantially reduced or perhaps completely eliminated through the following steps [18-20].

(a) Individual channel power reduction

(b) Increased dispersion (Phase Mismatch)

(c) Increased channel spacing.

In a multi wavelength system like DWDM, three waves mix together and produces the fourth wave given by Equation (3). 
$P=\frac{\left(D X l_{e f f}\right)^{2}}{A_{e f f}^{2}} \frac{1024 \pi^{6}}{n^{4} c^{2} \lambda^{2}} P_{i} P_{j} P_{K} e^{-\alpha L} \eta$

Equ.3 [16]

Where

$\mathrm{n}$ is the refractive index

$\lambda \& c$ are the wavelength and speed of the light respectively.

$P_{i} P_{j} P_{K}$ are the input powers of the three channels.

$\mathrm{X}$ is the electric susceptibility.

D is the degeneracy factor which is 3 for Two tone and 6 for Three tone mixing.

$\eta$ is the Four wave mixing efficiency (inversely proportional to Dispersion).

\section{iv. FWM FOR EQUAL AND UNEQUAL CHANNEL SPACING}

From Figure 2, it is inferred that when the inter channel spacing is equal means then the FWM power falls on the original signals such that it will induce crosstalk. To avoid this, unequally spaced channels are used. Fabrizio (1995) has analysed the FWM for different bandwidth expansion factors and demonstrated the reduction in overlapping of interfering FWM terms with the original channels for the unequal channel spacing [16-20].

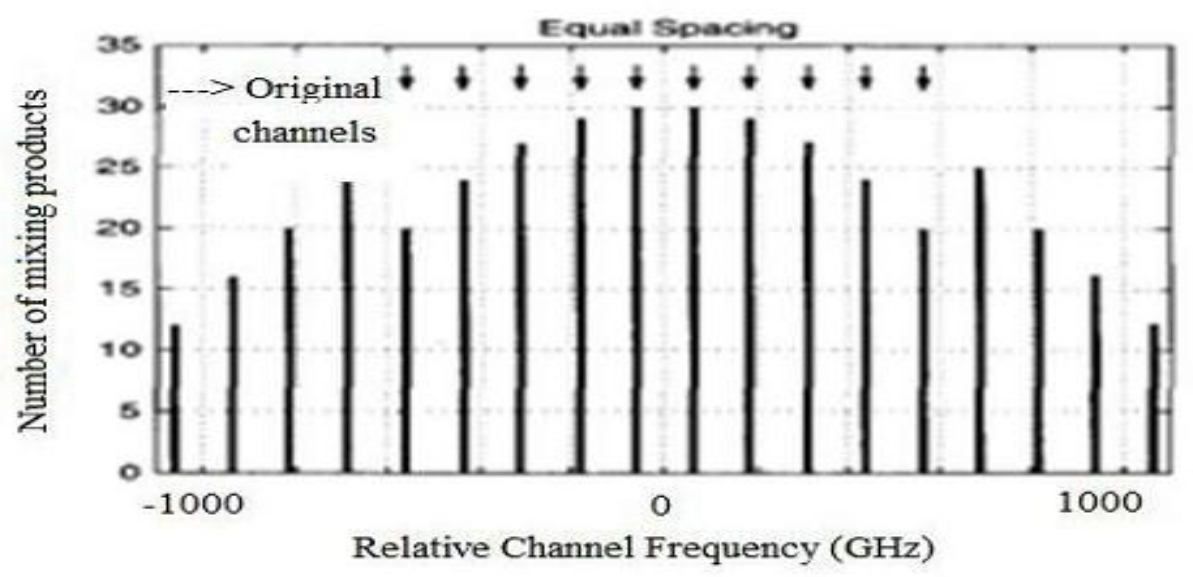

Figure .2: Spectrum for Equally spaced channels.

[Courtesy : FabrizioForghieri,JLWT,Vol.13,No.5, Page:891,1995] 


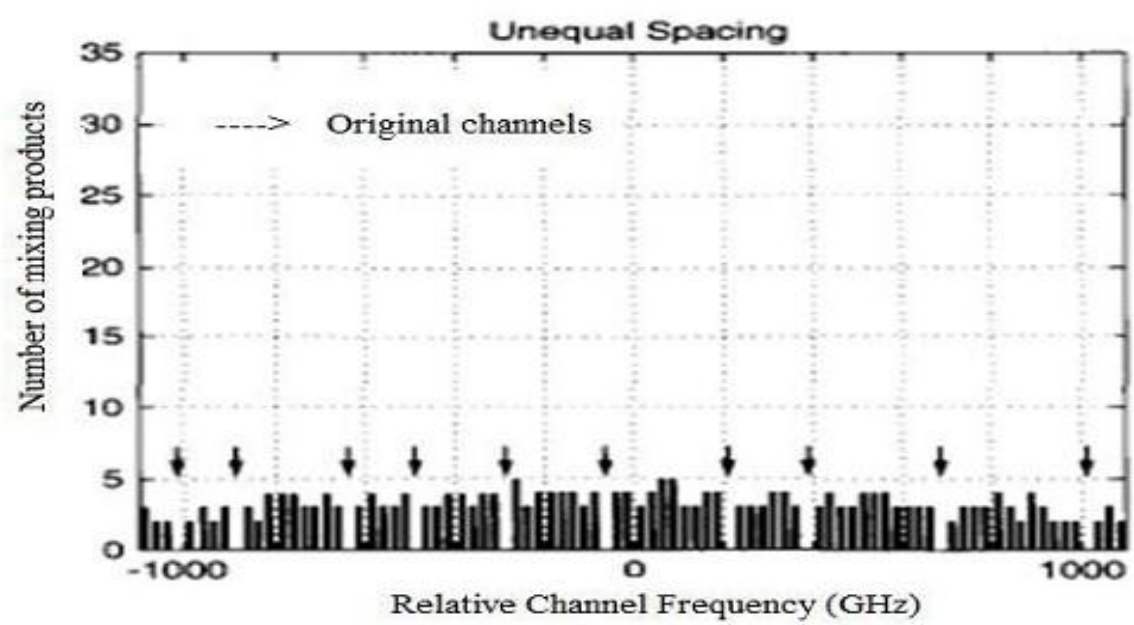

Figure.3: Spectrum for unequally spaced channels

[Courtesy: Fabrizio Forghieri , JLWT, Vol.13, No.5, Page: 891, 1995].

The FWM effect is described as [12] :

$$
\left(\nabla^{2}+k_{q}^{2}\right) E_{q}=-S_{q} \quad q=1,2,3,4 \quad \text { Equ.4 }
$$

In order to fulfill the coupling conditions, the four frequencies must be commensurate in a manner of $\omega_{1}, \omega_{2}, \omega_{3}$, and $\omega_{4}$, see Fig. (3).

Combining three waves, a fourth wave can be generated and the four wave signals are written as [12]:

$\frac{\partial A_{1}}{\partial_{Z}}=-\frac{\alpha}{2} A_{1}+j \gamma\left(\left|A_{1}\right|^{2}+C\left|A_{2}\right|^{2}+2\left|A_{3}\right|^{2}+C\left|A_{4}\right|^{2}\right) A_{1}+j C \gamma A_{2} A_{3} A_{4}^{*} \exp (j \Delta \beta Z)$. Equ.5

$\frac{\partial A_{2}}{\partial_{Z}}=-\frac{\alpha}{2} A_{2}+j \gamma\left(C\left|A_{1}\right|^{2}+\left|A_{2}\right|^{2}+C\left|A_{3}\right|^{2}+2\left|A_{4}\right|^{2}\right) A_{2}+j C \gamma A_{1} A_{3}^{*} A_{4} \exp (j \Delta \beta Z)$. Equ. 6

$\frac{\partial A_{3}}{\partial_{Z}}=-\frac{\alpha}{2} A_{3}+j \gamma\left(2\left|A_{1}\right|^{2}+C\left|A_{2}\right|^{2}+\left|A_{3}\right|^{2}+C\left|A_{4}\right|^{2}\right) A_{3}+j C \gamma A_{1} A_{2}^{*} A_{4} \exp (j \Delta \beta Z)$.Equ.7

$\frac{\partial A_{4}}{\partial_{Z}}=-\frac{\alpha}{2} A_{4}+j \gamma\left(C\left|A_{1}\right|^{2}+2\left|A_{2}\right|^{2}+C\left|A_{3}\right|^{2}+\left|A_{4}\right|^{2}\right) A_{4}+j C \gamma A_{1}^{*} A_{2} A_{3} \exp (j \Delta \beta Z)$. Equ.8

Where $A_{j}$ is the slowly varying envelope amplitude of the optical field with frequency $\omega_{j}, \alpha$ is the fiber loss coefficient and $\gamma$ is the nonlinear coef ficient. Depending on the state of polarization of the waves, the parameter $\mathrm{C}=2$ for parallel polarization and $\mathrm{C}=2 / 3$ for orthogonal polarization. The propagation mismatch constant $\Delta \beta=\left(\beta_{1}+\beta_{4}\right)-\left(\beta_{2}+\beta_{3}\right)$, where $\beta_{j}=\beta\left(\omega_{j}\right)(j=1,2,3$ and 4$)$ are the propagation constants in the fiber. 


\section{v. EFFECT OF DISPERSION ON FWM}

The dispersion in the fiber produces the phase mismatch and hence the interfering terms may get reduced. The simulation of the layout in the optsim software gives the power of the FWM terms for various no. of channels [20-22]. The dispersion in the fiber could be set from $0 \mathrm{ps} / \mathrm{nm} / \mathrm{km}$ to any value $[15,16,20]$.

Table 4.1 FWM Vs No. of Channels for the Dispersion of $0 \mathrm{ps} / \mathrm{nm} / \mathrm{km}$

\begin{tabular}{|r|r|r|r|r|r|}
\hline $\begin{array}{r}\text { No of } \\
\text { channels }\end{array}$ & $\begin{array}{r}\text { Input } \\
\text { frequency } \\
(\mathrm{THZ})\end{array}$ & $\begin{array}{r}\text { Input power } \\
(\mathrm{Mw})\end{array}$ & $\begin{array}{l}\text { FWM power } \\
(\mathrm{MW})\end{array}$ & $\begin{array}{r}\text { OSNR (dBm) } \\
\text { Frequency at } \\
\text { which } \\
\text { Max. FWM } \\
\text { occurs } \\
((\mathrm{THz}\end{array}$ \\
\hline 1 & 192 & 1 & 0.0000 & $\infty$ & $\begin{array}{r}\text { No } \\
\hline 2\end{array}$ \\
\hline 8 & $192 \& 192.2$ & 1 & 0.0400 & 13.98 & 192.2 \\
\hline 16 & {$[192: 0.2: 193.4]$} & 1 & 0.0510 & 12.92 & 193.0 \\
\hline 32 & {$[188: 0.2: 195]$} & 1 & 0.0511 & 12.91 & 195.0 \\
\hline 64 & $185: 0.2: 198.6$ & 1 & 0.0520 & 12.84 & 196.0 \\
\hline & & 1 & 0.0560 & 12.51 & 203.4 \\
\hline
\end{tabular}

\section{vi. Minimization of FWM Effects}

Traditional non-multiplexed systems have used dispersion shifted fiber at $1550 \mathrm{~nm}$ to reduce chromatic dispersion. Unfortunately operating at the dispersion minimum increases the level of FWM. Conventional fiber (dispersion minimum at $1330 \mathrm{~nm}$ ) suffers less from FWM but chromatic dispersion rises. Solution is to use "Non-Zero Dispersion Shifted Fiber" (NZ DSF), a compromise between DSF and conventional fiber (NDSF, Non-DSF). ITU-T standard is G.655 for non-zero dispersion shifted single mode fibers. By using unequal spacing between DWDM channels effect of FWM decreases [14-16, 22-25].

vii. PROGRAMS FOR FINDING OUT THE EXACT NUMBER OF FWM INTERFERING TERMS

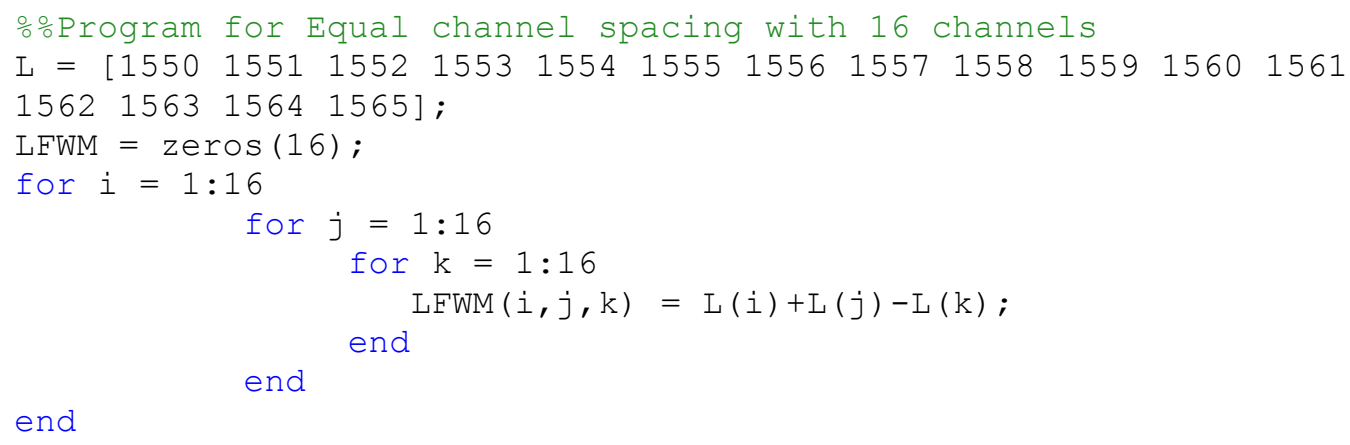




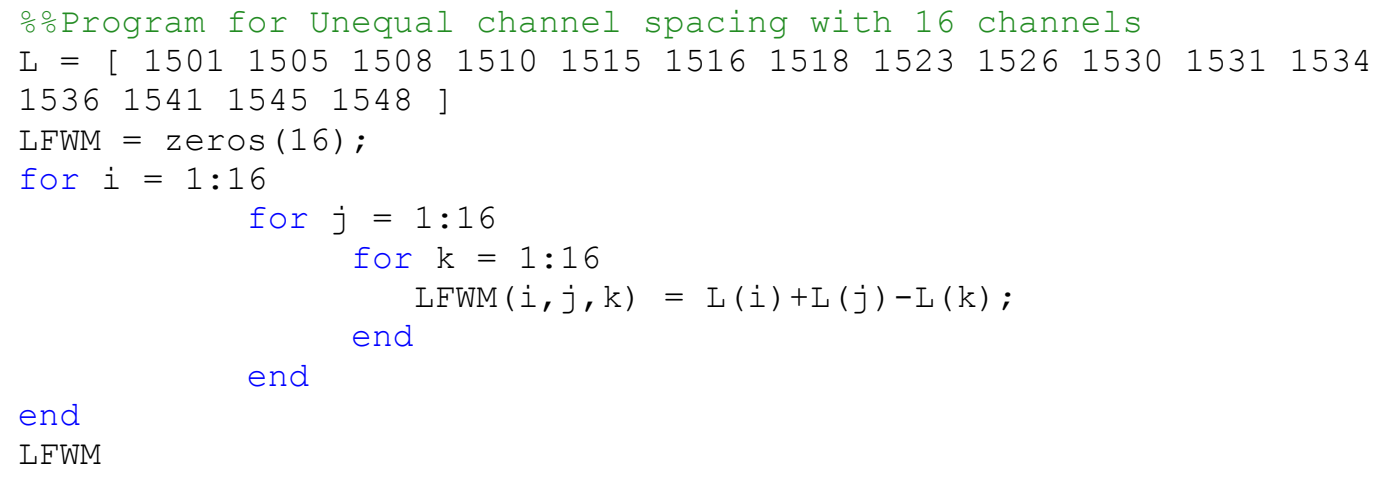




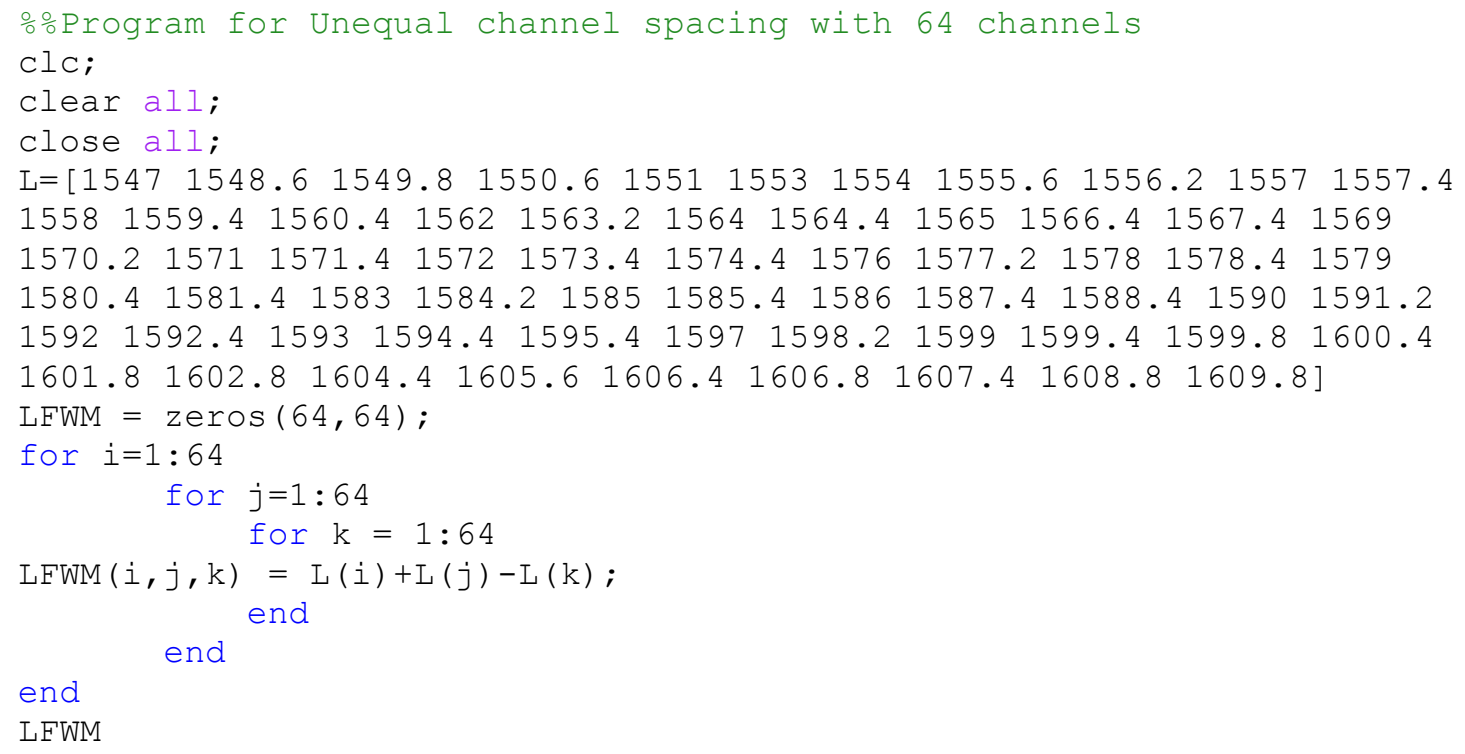

The Matlab programs are executed and the interfering terms are Table 2.

\begin{tabular}{|l|l|l|l|l|}
\hline $\begin{array}{l}\text { Number of } \\
\text { Channels }\end{array}$ & $\begin{array}{l}\text { Equal } \\
\text { Channel } \\
\text { Spacing }\end{array}$ & $\begin{array}{l}\text { Unequal Channel } \\
\text { Spacing [Bandwidth } \\
\text { Expansion Factor = 1] }\end{array}$ & $\begin{array}{l}\text { Unequal Channel } \\
\text { Spacing [Bandwidth } \\
\text { Expansion Factor = 1.5] }\end{array}$ & $\begin{array}{l}\text { Unequal Channel } \\
\text { Spacing[Bandwidth } \\
\text { Expansion Factor = 2 }\end{array}$ \\
\hline 4 & 104 & 92 & 78 & 56 \\
\hline 8 & 344 & 296 & 245 & 203 \\
\hline 16 & 11762 & 7984 & 5678 & 4548 \\
\hline 32 & 21844 & 13009 & 11232 & 9459 \\
\hline 64 & 39720 & 22815 & 20345 & 18367 \\
\hline
\end{tabular}

Table 2. Comparison of Interfering Terms.

The numbers inside the cells of the table are obtained by counting the coinciding terms for each channel from the results of execution of Matlab program.

\section{viii. RSULTS AND DISCUSSION}

By varying the dispersion from 0 to $4 \mathrm{ps} / \mathrm{nm} / \mathrm{km}$ we observed effect of dispersion on FWM by Optisystem 7. And also effect equal and unequal spacing on FWM is observed. These effects are shown in following figs (5) and (6) [15] . 
를 Optical Spectrum Analyzer

Dbl Click On Objects to open properties. Move Objects with Mouse Drag

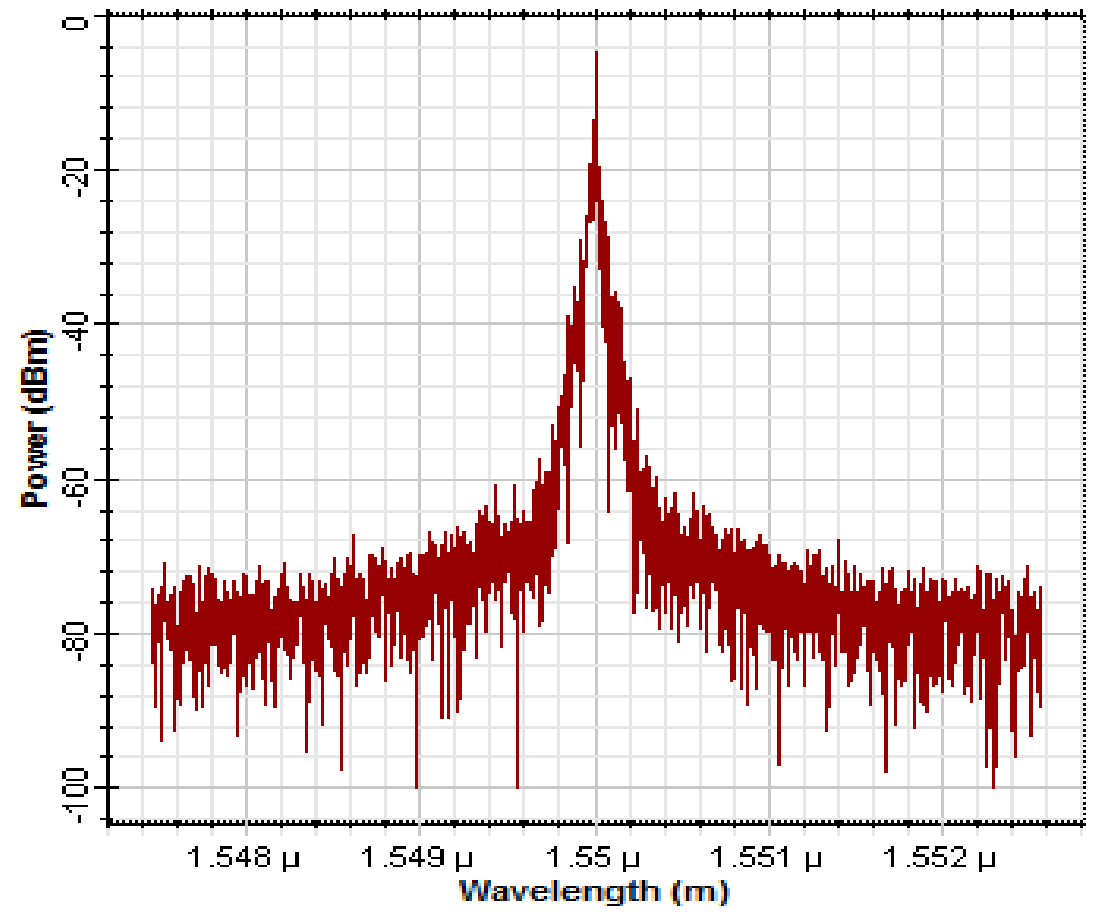

Fig.4: The spectrum at the fiber end

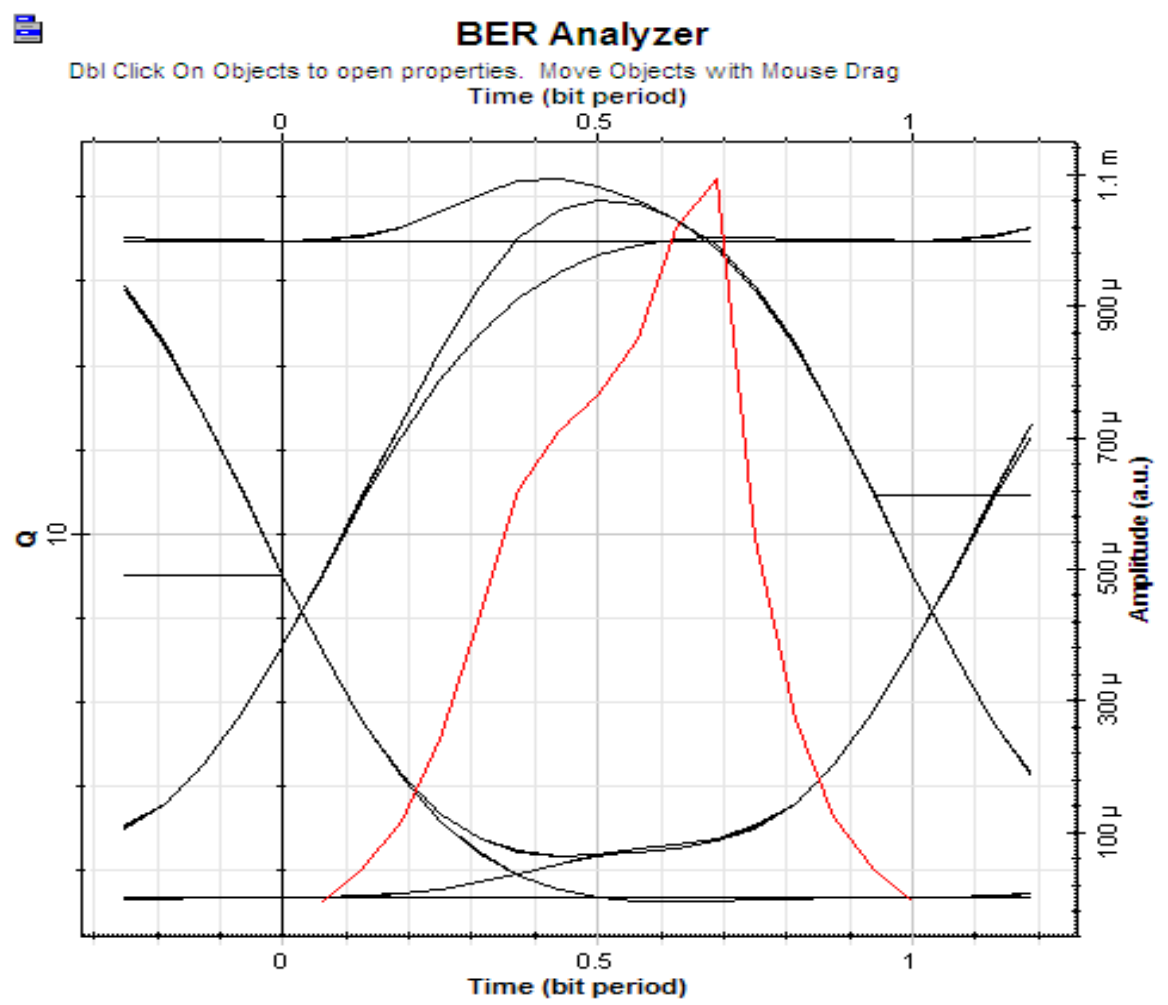

Fig.5: Eye Spectrum. 
를 Optical Time Domain Visualizer_1

Dbl Click On Objects to open properties. Move Objects with Mouse Drag

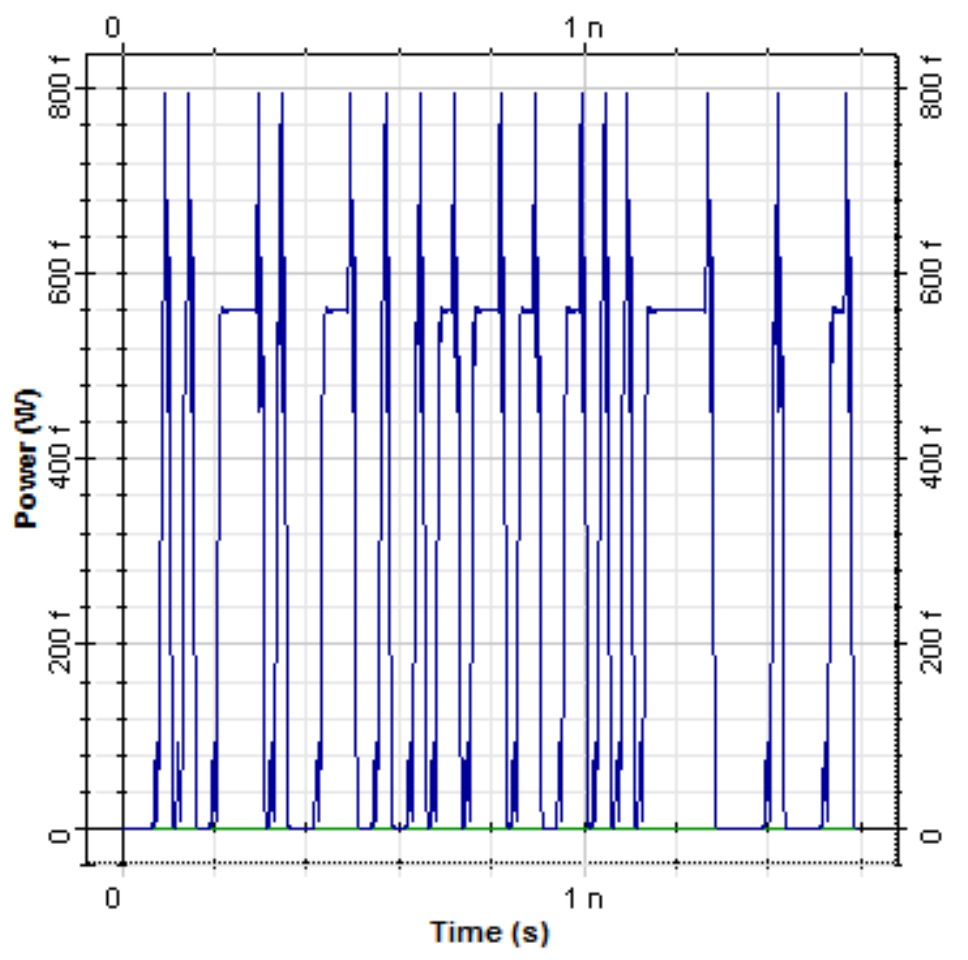

Fig.6: Optical Time Domain Visualizer, Time(s) vs Power (W) .

\section{ix. CONCLUSION}

FWM leads to interchannel crosstalk in DWDM systems. It generates additional noise and degrades system performance. By using non zero dispersion shifted fiber i.e. fiber having $4 \mathrm{ps} / \mathrm{nm} / \mathrm{km}$ and using unequal spacing among channels FWM effect can be reduced. As Unequal channel spacing could simultaneously reduce non linearities [FWM \& SRS], it may result in better performance reduced BER arising out of more OSNR.

\section{x. REFERENCES}

[1] Moncef B.Tayahi*, Sivakumar Lanka, Banmali Rawat, Four Wave Mixing in Closely Spaced DWDM OpticalChannels, International Journal of Microwave and Optical Technology,vol.1, No.2.August 2006.

[2] J. Toulouse, Optical Nonlinearities in Fibers: Review, Recent Examples, and Systems Applications, Journal of Light Wave Technology, Vol. 23, No. 11,November 2005.

[3] L. G. L. Wegener, M. L. Povinelli, A. G. Green, P. P. Mitra, J. B. Stark, P. B. Littlewood, The effect of propagating nonlinearities on the information capacity of 
WDM optical fiber systems: Cross-phase modulation and four-wave mixing, Physica, D, vol. 189, no. 1-2, pp. 81-99,Feb. 15, 2004.

[4]. N. Bloembergen, editor, Proceedings of the International School of Physics Enrico Fermi Course LXIV: Nonlinear Spectroscopy, (North Holland, Amsterdam, 1977) .

[5]. N. Bloembergen, Nonlinear Optics, (World Scientific Publishing, Singapore, 1996)

[6]. N. Bloembergen, "Nonlinear Optics and Spectroscopy," Science 216, 1057-1064 (1982).

[7] M.C.GuptaandJ.Ballato, "The hand book of photonics," $2^{\text {nd }}$ Edition,CRC Press, 2006.

[8] G.P.Agrawal,“Fiber-optic communication systems,”4th Edition,Wiley,2010.

[9] A. Chowdhury, C .Staus, B . F . Boland, T . F .Kuech, and L . Mc Caughan, "Experimental demonstration of 1535:1555 nm simultaneous optical wavelength interchange with a nonlinear photonic crystal,"Opt. Lett., vol.26, no.17,pp. 13531355 ,Sep.2001.

[11] K.Uesaka,K.K.-Y.Wong,M.E.Marhic,andL.G.Kazovsky, "WaveLength exchange in a highly non linear dispersion- shifted fiber : Theory and experiments," IEEE J . Sel.Topics Quantum Electron.,vo I. 8,no.3, pp.560568,May/Jun.2002.

[12]. Performance Evaluation of Wavelength Exchanging in Optical Interconnect Tawfik Ismail ,Hassan Mostafa ,and Yehia Ismail, 978-1-5090-0246-7/15/\$31.00 C2015 IEEE.

[13] G. P. Agrawal, Applications of Nonlinear Fiber Optics, (San Diego, CA : Academic, 2001).

[14] Introducing DWDM

http://www.cisco.com/univercd/cc/td/doc/product/mels/dwdm/dwdm fns.htm

[15] Four Wave Mixing In DWDM Optical System, Gouri Deshmukh, Prof. Santosh Jagtap , International Journal of Computational Engineering Research ||Vol, 03 || Issue, 6||.

[16]. Analysis of Four Wave Mixing in Fiber Optic Communication and to devise a mechanism for combating the FWM', has been published in an International Conference on Emerging Trends in Engineering Technologies-2010, organized by Noorul Islam University, March 25-26, 2010.

[17] Hien T. T. Pham., Ngoc T. Dang: Performance improvement of spatial 
modulation-assisted FSO systems over Gamma-Gamma fading channels with geometric spreading, Photon Netw Commun, 34, pp.213-220, 2017

[18] E. E. Elsayed and B. B. Yousif, "Performance evaluation and enhancement of the modified OOK based IM/DD techniques for hybrid fiber/FSO communication over WDM-PON systems," Opt. Quantum Electron., vol. 52, no. 9, 2020, doi: 10.1007/s11082-020-02497-0.

[19] B. B. Yousif, E. E. Elsayed, and M. M. Alzalabani, "Atmospheric turbulence mitigation using spatial mode multiplexing and modified pulse position modulation in hybrid RF/FSO orbital-angular-momentum multiplexed based on MIMO wireless communications system," Opt. Commun., vol. 436, pp. 197208, 2019, doi: 10.1016/j.optcom.2018.12.034.

[20] A. M. Mbah, J. G. Walker, and A. J. Phillips, "Outage probability of WDM freespace optical systems affected by turbulence-accentuated interchannel crosstalk," IET Optoelectron., vol. 11, no. 3, pp. 91-97, 2017, doi: 10.1049/ietopt.2016.0057.

[21] B. B. Yousif and E. E. Elsayed, "Performance Enhancement of an OrbitalAngular-Momentum-Multiplexed Free-Space Optical Link under Atmospheric Turbulence Effects Using Spatial-Mode Multiplexing and Hybrid Diversity Based on Adaptive MIMO Equalization," IEEE Access, vol. 7, pp. 84401-84412, 2019, doi: 10.1109/ACCESS.2019.2924531.

[22] E. E. Elsayed and B. B. Yousif, "Performance enhancement of hybrid diversity for $\mathrm{M}$-ary modified pulse-position modulation and spatial modulation of MIMOFSO systems under the atmospheric turbulence effects with geometric spreading," Opt. Quantum Electron., vol. 52, no. 12, 2020, doi: 10.1007/s11082-020-02612-1.

[23] Ebrahim E. Elsayed, Bedir B. Yousif, and Mahmoud M. Alzalabani, "Performance enhancement of the power penalty in DWDM FSO communication using DPPM and OOK modulation", Optical and Quantum Electronics, vol. 50 (7), pp. 282, (26 June 2018).

[24] Ansari, N., Zhang, J.: 'Media access control and resource allocation for next generation passive optical networks' (Springer, 2013)

[25] Ebrahim E. Elsayed and Bedir B. Yousif, "Performance enhancement of the average spectral efficiency using an aperture averaging and spatial-coherence diversity based on the modified-PPM modulation for MISO FSO links", Optics Communications, vol. 463, pp. 125463, (15 May 2020).

\section{Compliance with ethical standards}

Conflict of interest: The author declares that there is no conflict of interest regarding the manuscript. The author is responsible for the content and writing of this article. The author declares that he has no known competing financial interests or personal relationships that could have appeared to influence the work reported in this paper. 